\title{
Resonant Charge Exchange in Atom Scattering on Atoms Adsorbed on Metal Surfaces
}

\author{
M. Wiertel, E. TARANko And R. TARANKo* \\ Institute of Physics, M. Curie-Skłodowska University \\ pl. M. Curie-Skłodowskiej 1, 20-031 Lublin, Poland
}

(Received June 1, 2001; revised version March 8, 2002)

\begin{abstract}
The ionization probability of an atom scattered from an atom adsorbed on a metal surface has been studied theoretically within the time-dependent Anderson-Newns model. The effect of the metal electron density of states, the band filling as well as the relative position of the scattered atom and adsorbed atom energy levels have been considered and the comparison with the results obtained for clean surfaces has been made.
\end{abstract}

PACS numbers: 79.20.-m, 82.65.+r, 34.70.+e

\section{Introduction}

The interaction of ions/atoms with surfaces is a very complex process involving many fundamental phenomena. One of such phenomena is a resonant charge transfer (RCT) between the incident ions/atoms and the target surfaces. This process, when energetically possible, is very efficient and plays a key role in many dynamical processes at surfaces. Understanding this typically nonadiabatic phenomenon is of primary importance for description of many surface analysis techniques, e.g. secondary-ion mass spectroscopy or ion-scattering spectroscopy.

The resonance tunneling of electrons between the moving atom and the conduction band of the metal surface is conventionally described in the framework of the time-dependent Anderson-Newns (AN) model (e.g. [1-13]). The motion of the ion/atom centre of mass can be treated classically, hence, the position de-

*corresponding author; e-mail: taranko@tytan.umcs.lublin.pl 
pendence of the parameters, such as the energy of ionization and affinity levels, the hybridization matrix elements and the Coulomb intraatomic electron-electron interaction can be easily converted into an explicit time dependence. The calculations of the electron occupancy of atoms backscattered from clean surfaces have been the subject of many papers. Most of them were made within the wide band limit (WBL) (see, e.g. [7-9]). Some calculations for atom occupancy were made for one-dimensional models (e.g. [4, 14, 15]), for cluster tight-binding models (e.g. [16]) or within the approach enabling us to introduce the realistic surface electronic structure (e.g. $[1,2,17,18]$ ).

The AN model was able to give a reasonable explanation of many experimental findings, e.g. characteristic oscillation of the neutralization probability of $\mathrm{He}^{+}$ ions scattered on metal surfaces as a function of the inverse ion velocity [19], the exponential dependence upon the inverse atom velocity of the fraction of $\mathrm{Li}^{-}$ions after scattering on a caesiated W(110) surface [20], the work function dependence of the probabilities of charged states (of being negative, positive or neutral) of $\mathrm{Na}$ atoms scattered on metal surfaces and increasing the probability of being neutral with the increasing temperature of sodium atoms scattered on W(100) [21, 22].

The above-mentioned studies considered clean and perfect surfaces. The main surface characteristic was the work function and the surface electron density of states. However, realistic surfaces have adsorbed impurities which can influence the charge transfer between the surface and the scattered atom. There is much experimental evidence that the presence of additional atoms at the surface changes the process of the charge transfer [23], e.g. the presence of oxygen at the surface of silicon strongly enhances the ionization probability of sputtered Si atoms [16]. Usually, the adsorption of atoms on metal surfaces results in a change of the work function. The work function is a global characteristics of the surface and enters the theoretical expressions for electron occupancy of the sputtered or scattered atoms. However, in order to describe many experimental findings on the ionization or neutralization of atoms scattered (sputtered) on many contaminated surfaces one has to take into consideration also the changes of the surface local electrostatic potential around the adsorbed atom.

For example, the analysis of the data on low-energy positive alkali ions scattered on caesiated $\mathrm{Cu}(100)$ surface indicates that the area around the adsorbate will produce a higher neutralization probability of scattered ions than other places of the surface [24].

The resonant charge transfer process is sensitive to whether the atom is backscattered from the adsorbate or from the substrate atoms, e.g. see the results of Weare and Yarmoff [25] on the $\mathrm{Li}^{+}$backscattering from the alkali-covered $\mathrm{Al}(100)$ surface. The experiment is able to measure the electron occupancy of atoms scattered from a given adsorbed atom or from a substrate atom [e.g. 23, 25-27]. Therefore, it should be interesting to investigate theoretically the charge transfer process between the scattered atom and the surface through the adsorbed atom. In such 
a case, the electron can be (additionally) transferred from the scattering atom to the surface energy band through the adsorbed atom level. The final charge state of the backscattered atom should depend on the relative position of both atom energy levels and Fermi level, as well as on the energy of the scattered atom and on the strength of interaction between adatom, surface and scattered atom. In this paper such investigations are reported and can be regarded as generalization of the results of Tsuneyuki et al. [1] and of Tsukada [2] to the case of scattering on atoms adsorbed on metal surfaces and the results of Kato et al. [6] to the surfaces with the arbitrary electronic structure. In addition, in order to come closer to realistic situations of atoms scattering on transition metal substrates, we have performed calculations of the negative ionization probability of atoms scattered on clean substrates described by the electronic band structure consisting of overlapped broad and narrow energy bands for various configurations of these bands and other parameters describing this process.

The paper is arranged as follows. In Sec. 2 the model is defined. In Sec. 3 the calculations of the electron occupancy of the scattered atom are given and Sec. 4 includes numerical results and conclusions. We use the atomic units (a.u.) of the time, distance, and velocity.

\section{Model of the electron transfer}

We use the independent particle model described by the Hamiltonian (for simplicity, the spin variables are neglected)

$$
\begin{aligned}
H= & \sum_{\boldsymbol{k}} \varepsilon_{k} c_{\boldsymbol{k}}^{+} c_{\boldsymbol{k}}+\varepsilon_{\mathrm{A}} c_{\mathrm{A}}^{+} c_{\mathrm{A}}+\varepsilon_{0} c_{0}^{+} c_{0}+\sum_{\boldsymbol{k}}\left(V_{\boldsymbol{k}} c_{\boldsymbol{k}}^{+} c_{\mathrm{A}}+\text { h.c. }\right) \\
& +V_{\mathrm{A} 0}(t) c_{\mathrm{A}}^{+} c_{0}+\text { h.c. }
\end{aligned}
$$

where $c_{\mathrm{A}}, c_{0}, c_{k}\left(c_{\mathrm{A}}^{+}, c_{0}^{+}, c_{k}^{+}\right)$are the electron annihilation (creation) operators for the adsorbed atom orbital, the incident atom orbital and the surface electron state orbital. The functions $V_{\mathrm{A} 0}(t)$ and $V_{\boldsymbol{k}}$ are the matrix elements of interaction between the moving (scattered) and adsorbed atoms and between the adsorbed atom and $k$-th level of the surface electron band, respectively. The incident atom (labelled by a number " 0 ") and the adsorbed atom (labelled by a letter " $\mathrm{A}$ ") are characterized by its valence electron levels $\varepsilon_{\mathrm{A}}$ and $\varepsilon_{0}(t)$ and the dispersion of the surface electron band is described by $\varepsilon_{k}$. The corresponding electron wave functions are denoted as $|0\rangle,|a\rangle$, and $|k\rangle$, respectively. The centre of mass of the atom is assumed to move along the classical trajectory perpendicular to the surface.

In our model we ignore the RCT between the incident atom and the substrate conduction band. This assumption can be justified as we consider a head-on collision with a single adsorbed atom, i.e. the collisional atom approaches the surface along the surface normal above an adsorbed atom. As the charge transfer between the substrate surface and moving atom is a strongly $z$-depending function, $z$ being 
the distance between the surface and scattered atom, then, in the first approximation, this channel of the charge transfer can be ignored. The behaviour of $\varepsilon_{0}$ with the distance $z$ from the surface is uncertain in detail though it is expected to be equal to the affinity level at large $z$, and to decrease as $z$ decreases due to image effects. For simplicity, we assume $\varepsilon_{0}$ as independent of $z$ and only the $z$-dependence of $V_{\mathrm{A} 0}(z)$ is taken into consideration, as this dependence should influence more the resulting charge transfer than the changes of $\varepsilon_{0}$. In literature various forms of the $z$-dependence of the hopping integral between atoms and surface have been explored - exponential, Gaussian, nodal or other forms [2, 7, 14, 21]. Usually used the exponential form fairly well represents the true hopping integral in the region far away from the surface but it is overestimated at distances close to the surface. For that reason it is convenient to choose a Gaussian form for $z$-dependence of $V_{\mathrm{A} 0}(z)$ according to a simple formula [9] although other parametrization are also used

$$
V_{\mathrm{A} 0}(z)=V_{\mathrm{A} 0} f(z)=V_{\mathrm{A} 0} \exp \left(-\gamma z^{2} / 2\right),
$$

where $\gamma$ characterizes the interaction range and usually is of the order of 0.05 a.u. ${ }^{-2}$. In addition, we have also performed the calculations for widely accepted in model calculations the sudden switching on of the interactions and take $f(t)=0$ for $t \leq 0$ and $f(t)=1$ for $t>0$. In this case the results are independent of the atom velocity. Note that because the motion of the atom's centre of mass is treated classically and the energy and trajectory of the atom are known, the position dependence of the parameters can be easily transformed to $t$-dependence. As for the matrix element $V_{\mathrm{A} \boldsymbol{k}}$ (it should be time-independent) we adopt the sudden switching on of the interactions and take it in the following form:

$$
V_{\mathrm{A} \boldsymbol{k}}(z)=V_{\mathrm{A} \boldsymbol{k}} u(t),
$$

where $u(t)=0$ for $t \leq 0$ and $u(t)=1$ for $t>0$.

\section{Calculations of the charge transfer}

To calculate the occupancy of the moving atom we use the time-evolution operator technique [1-5]. The dynamical evolution of the system can be described in terms of the time-evolution operator $U\left(t, t_{0}\right)$ (in the interaction representation) given by the equation of motion [2]

$$
\mathrm{i} \frac{\partial}{\partial t} U\left(t, t_{0}\right)=\tilde{V}(t) U\left(t, t_{0}\right)
$$

and the condition $U\left(t_{0}, t_{0}\right)=1$, where

$$
\begin{aligned}
& \tilde{V}(t)=U_{0}\left(t, t_{0}\right) V(t) U_{0}^{+}\left(t, t_{0}\right), \\
& U_{0}\left(t, t_{0}\right)=\exp \left[\mathrm{i} H_{0}\left(t-t_{0}\right)\right] .
\end{aligned}
$$

Here, $H_{0}$ denotes the three first terms of $(1)$ and $V(t)$ is defined as 


$$
V(t)=\sum_{\boldsymbol{k}}\left(V_{\boldsymbol{k} \mathrm{A}} c_{\boldsymbol{k}}^{+} c_{\mathrm{A}}+\text { h.c. }\right)+V_{\mathrm{A} 0}(t) c_{\mathrm{A}}^{+} c_{0}+\text { h.c. }
$$

Once the time-evolution operator $U$ is found, the occupancy of the moving atom can be written as $[1-3]$

$$
n_{0}(t)=n_{0}\left(t_{0}\right)\left|U_{00}\left(t, t_{0}\right)\right|^{2}+n_{\mathrm{A}}\left(t_{0}\right)\left|U_{0 a}\left(t, t_{0}\right)\right|^{2}+\sum_{k} n_{\boldsymbol{k}}\left(t_{0}\right)\left|U_{0 \boldsymbol{k}}\left(t, t_{0}\right)\right|^{2} .
$$

Here $n_{0}\left(t_{0}\right), n_{\mathrm{A}}\left(t_{0}\right)$, and $n_{\boldsymbol{k}}\left(t_{0}\right)$ are the initial occupancies of moving atom, adsorbed atom, and substrate energy levels, respectively. $U_{i j}\left(t, t_{0}\right) \equiv\left\langle i\left|U\left(t, t_{0}\right)\right| j\right\rangle$ denote the appropriate matrix elements of the time-evolution operator $U$, where $|i\rangle$ and $|j\rangle$ belong to the set of basis function $|a\rangle,|0\rangle,|k\rangle$ and the identity $I=$ $|a\rangle\langle a|+| 0\rangle\left\langle 0\left|+\sum_{k}\right| k\right\rangle\langle k|$ was assumed.

To complete the specifications of our model we note that if we consider only one atom orbital, then $n_{0}(t)$ (Eq. (8)) corresponds to the probability of the negative ionization (the occupancy of the affinity level) if $\varepsilon_{0}$ denotes the energy of the affinity level. In the other case, if $\varepsilon_{0}$ describes the atom ionization level, then the probability of the positive ionization is given by $1-n_{0}(t)[5]$.

It follows from Eq. (8) that in order to calculate the occupancy of the moving atom backscattered from the atom adsorbed on the surface we have to know the functions $U_{00}\left(t, t_{0}\right), U_{0 a}\left(t, t_{0}\right)$, and $U_{0 \boldsymbol{k}}\left(t, t_{0}\right)$. Similarly in scattering on clean surfaces, one can construct the sets of integro-differential equations for each of these functions. In order to calculate $U_{00}\left(t, t_{0}\right)$ one should solve the following set of equations:

$$
\begin{aligned}
& \frac{\partial}{\partial t} U_{00}\left(t, t_{0}\right)=-\mathrm{i} \tilde{V}_{0 a}(t) U_{a 0}\left(t, t_{0}\right) \\
& \frac{\partial}{\partial t} U_{a 0}\left(t, t_{0}\right)=-\mathrm{i} \tilde{V}_{a 0}(t) U_{00}\left(t, t_{0}\right)-\mathrm{i} \sum_{q} \tilde{V}_{a q}(t) U_{q 0}\left(t, t_{0}\right) \\
& \frac{\partial}{\partial t} U_{\boldsymbol{q} 0}\left(t, t_{0}\right)=-\mathrm{i} \tilde{V}_{\boldsymbol{q} a}(t) U_{a 0}\left(t, t_{0}\right)
\end{aligned}
$$

where the matrix elements $\tilde{V}_{i j}(t)$ can be written as

$$
\tilde{V}_{i j}(t)=V_{i j}(t) \exp \left[\mathrm{i}\left(\varepsilon_{i}-\varepsilon_{j}\right) t\right]
$$

and $\varepsilon_{i}$ corresponds to $|i\rangle$.

Similarly, for $U_{0 a}\left(t, t_{0}\right)$ and $U_{0 \boldsymbol{k}}\left(t, t_{0}\right)$ the corresponding sets of equations should be solved:

$$
\begin{aligned}
& \frac{\partial}{\partial t} U_{0 a}\left(t, t_{0}\right)=-\mathrm{i} \tilde{V}_{0 a}(t) U_{a a}\left(t, t_{0}\right) \\
& \frac{\partial}{\partial t} U_{a a}\left(t, t_{0}\right)=-\mathrm{i} \tilde{V}_{a 0}(t) U_{0 a}\left(t, t_{0}\right)-\mathrm{i} \sum_{q} \tilde{V}_{a q}(t) U_{q a}\left(t, t_{0}\right),
\end{aligned}
$$




$$
\frac{\partial}{\partial t} U_{\boldsymbol{q} a}\left(t, t_{0}\right)=-\mathrm{i} \tilde{V}_{q a}(t) U_{a a}\left(t, t_{0}\right)
$$

and

$$
\begin{aligned}
& \frac{\partial}{\partial t} U_{0 \boldsymbol{q}}\left(t, t_{0}\right)=-\mathrm{i} \tilde{V}_{0 a}(t) U_{a \boldsymbol{q}}\left(t, t_{0}\right), \\
& \frac{\partial}{\partial t} U_{a \boldsymbol{k}}\left(t, t_{0}\right)=-\mathrm{i} \tilde{V}_{a 0}(t) U_{0 \boldsymbol{k}}\left(t, t_{0}\right)-\mathrm{i} \sum_{q} \tilde{V}_{a \boldsymbol{q}}(t) U_{\boldsymbol{q} \boldsymbol{k}}\left(t, t_{0}\right), \\
& \frac{\partial}{\partial t} U_{\boldsymbol{k} \boldsymbol{q}}\left(t, t_{0}\right)=-\mathrm{i} \tilde{V}_{\boldsymbol{k} a}(t) U_{a \boldsymbol{q}}\left(t, t_{0}\right),
\end{aligned}
$$

respectively.

Let us calculate the function $U_{00}\left(t, t_{0}\right)$. Writing the formal solutions of Eqs. (9) and (11) in the following form:

$$
\begin{aligned}
& U_{00}\left(t, t_{0}\right)=1-\mathrm{i} \int_{t_{0}}^{t} \mathrm{~d} t^{\prime} \tilde{V}_{0 a}\left(t^{\prime}\right) U_{a 0}\left(t^{\prime}, t_{0}\right), \\
& U_{q 0}\left(t, t_{0}\right)=-\mathrm{i} \int_{t_{0}}^{t} \mathrm{~d} t^{\prime} \tilde{V}_{q a}\left(t^{\prime}\right) U_{a 0}\left(t^{\prime}, t_{0}\right),
\end{aligned}
$$

and inserting them into Eq. (10) we obtain the integro-differential equation for $U_{a 0}\left(t, t_{0}\right)$

$$
\frac{\partial U_{a 0}\left(t, t_{0}\right)}{\partial t}=-\mathrm{i} V_{\mathrm{A} 0}(t) \exp \left[\mathrm{i}\left(\varepsilon_{\mathrm{A}}-\varepsilon_{0}\right)\left(t-t_{0}\right)\right]-\int_{t_{0}}^{t} \mathrm{~d} t^{\prime} \phi\left(t, t^{\prime}\right) U_{a 0}\left(t^{\prime}, t_{0}\right),
$$

where

$$
\begin{gathered}
\phi\left(t, t^{\prime}\right)=\left\{V_{\mathrm{A} 0}(t) V_{0 \mathrm{~A}}\left(t^{\prime}\right) \exp \left[\mathrm{i}\left(\varepsilon_{\mathrm{A}}-\varepsilon_{0}\right)\left(t-t^{\prime}\right)\right]\right. \\
\left.+\left|V_{\mathrm{A} k}\right|^{2} \mathcal{D}\left(t-t^{\prime}\right) \exp \left[\mathrm{i} \varepsilon_{\mathrm{A}}\left(t-t^{\prime}\right)\right]\right\} .
\end{gathered}
$$

Solving Eq. (21) (numerically) and inserting its solutions into Eq. (19) give the required function $U_{00}\left(t, t_{0}\right)$.

Similarly,

$$
\begin{aligned}
& U_{0 a}\left(t, t_{0}\right)=-\int_{t_{0}}^{t} \mathrm{~d} t^{\prime} \tilde{V}_{0 a}\left(t^{\prime}\right) U_{a a}\left(t^{\prime}, t_{0}\right), \\
& U_{0 \boldsymbol{k}}\left(t, t_{0}\right)=-\int_{t_{0}}^{t} \mathrm{~d} t^{\prime} \tilde{V}_{0 a}\left(t^{\prime}\right) U_{a \boldsymbol{k}}\left(t^{\prime}, t_{0}\right),
\end{aligned}
$$

where $U_{a a}\left(t, t_{0}\right)$ and $U_{a \boldsymbol{k}}\left(t, t_{0}\right)$ satisfy the integro-differential equations

$$
\frac{\partial}{\partial t} U_{a a}\left(t, t_{0}\right)=-\int_{t_{0}}^{t} \mathrm{~d} t^{\prime} \phi\left(t, t^{\prime}\right) U_{a a}\left(t^{\prime}, t_{0}\right)
$$




$$
\begin{gathered}
\frac{\partial}{\partial t} U_{a \boldsymbol{k}}\left(t, t_{0}\right)=-\mathrm{i} V_{\mathrm{A} \boldsymbol{k}}(t) \exp \left[\mathrm{i}\left(\varepsilon_{a}-\varepsilon_{\boldsymbol{k}}\right)\left(t-t_{0}\right)\right] \\
-\int_{t_{0}}^{t} \mathrm{~d} t^{\prime} \phi\left(t, t^{\prime}\right) U_{a \boldsymbol{k}}\left(t^{\prime}, t_{0}\right)
\end{gathered}
$$

respectively.

For arbitrary substrate density of states and $V_{\mathrm{A} 0}(t)$ Eqs. (16), (24), and (26) have to be solved by numerical methods and the results of such calculations will be presented in the next section. We want to stress that the model described by Eq. (1), if appropriately modified, allows us to obtain results for resonant charge exchange between two atoms in the absence of the surface. In such a case one should obtain probability that an electron initially placed, say, on one atom has hopped on the second atom, in the form of oscillations periodic in $1 / v, v$ being the relative velocity of atoms [19]. This process can be described by Eqs. (13, 14) (now $\tilde{V}_{a q}(t)=0$ )

$$
\begin{aligned}
& \frac{\partial U_{a a}\left(t, t_{0}\right)}{\partial t}=-\mathrm{i} \tilde{V}_{a 0}(t) U_{0 a}\left(t, t_{0}\right), \\
& \frac{\partial U_{0 a}\left(t, t_{0}\right)}{\partial t}=-\mathrm{i} \tilde{V}_{0 a}(t) U_{a a}\left(t, t_{0}\right) .
\end{aligned}
$$

If we assume that at $t=-\infty$ the electron was localized on " $\mathrm{A}$ "-atom and the " 0 " atom orbital was empty, then the wanted probability reads

$$
n_{0}(t)=n_{\mathrm{A}}(-\infty)\left|U_{0 a}(t,-\infty)\right|^{2} .
$$

After formal integration of Eq. (28) and insertion its solution into Eq. (27) we have

$$
\frac{\partial U_{a a}(t,-\infty)}{\partial t}=-\mathrm{i} \tilde{V}_{0 a}(t) \int_{-\infty}^{t} \mathrm{~d} t^{\prime} \tilde{V}_{a 0}\left(t^{\prime}\right) U_{a a}\left(t^{\prime},-\infty\right),
$$

which, for example, for $\varepsilon_{\mathrm{A}}=\varepsilon_{0}=0$ has the solution

$$
U_{a a}(t,-\infty)=\cos \int_{-\infty}^{t} \mathrm{~d} t^{\prime} V_{0 \mathrm{~A}}\left(t^{\prime}\right)
$$

At the same time we obtain

$$
U_{0 a}(t,-\infty)=-\mathrm{i} \sin \int_{\infty}^{t} \mathrm{~d} t^{\prime} V_{0 \mathrm{~A}}\left(t^{\prime}\right)
$$

and for $n_{\mathrm{A}}(\infty)$ we have a well-known solution

$$
n_{\mathrm{A}}(\infty)=\sin ^{2} \int_{-\infty}^{\infty} \mathrm{d} t^{\prime} V_{0 \mathrm{~A}}\left(t^{\prime}\right)
$$


If one assumes a constant atom velocity and the time-dependent perturbation $V_{0 \mathrm{~A}}(t)$, which is mostly different from zero only for small distances between colliding atoms, then the result (33) can be easily transformed to the form showing oscillations in $1 / v$.

At the end of this section we consider the occupancy of the moving atom scattered on a clean surface which is characterized by two overlapping energy bands. In this manner we want to mimic the real situations of scattering on transition metal substrates with one broad $s$-electron and narrow $d$-electron energy bands. In this case $n_{0}(t)$ can be written in the following form:

$$
\begin{aligned}
n_{0}(t) & =n_{0}\left(t_{0}\right)\left|U_{00}\left(t, t_{0}\right)\right|^{2}+\sum_{k_{s}} n_{\boldsymbol{k}_{s}}\left(t_{0}\right)\left|U_{0 \boldsymbol{k}_{s}}\left(t, t_{0}\right)\right|^{2} \\
& +\sum_{\boldsymbol{k}_{d}} n_{\boldsymbol{k}_{d}}\left(t_{0}\right)\left|U_{0 \boldsymbol{k}_{d}}\left(t, t_{0}\right)\right|^{2}
\end{aligned}
$$

where $\boldsymbol{k}_{s}, \boldsymbol{k}_{d}$ correspond to $s$ - and $\boldsymbol{d}$-electron energy bands, respectively. The integro-differential equations for required functions $U_{00}\left(t, t_{0}\right), U_{0 \boldsymbol{k}_{\mathrm{s}}}\left(t, t_{0}\right)$, and $U_{0 \boldsymbol{k}_{d}}\left(t, t_{0}\right)$ are similar to Eqs. $(25,26)$ and read

$$
\begin{aligned}
& \frac{\partial U_{00}\left(t, t_{0}\right)}{\partial t}=-\int_{t_{0}}^{t} d t^{\prime} \mathcal{K}\left(t, t^{\prime}\right) U_{00}\left(t^{\prime}, t_{0}\right) \\
& \frac{\partial U_{0 \boldsymbol{k}_{i}}\left(t, t_{0}\right)}{\partial t}=-\mathrm{i} V_{0 k_{i}}(t) \exp \left\{\mathrm{i} \int_{t_{0}}^{t} \mathrm{~d} t^{\prime}\left[\varepsilon_{0}\left(t^{\prime}\right)-\varepsilon_{\boldsymbol{k}_{i}}\right]\right\} \\
& -\int_{t_{0}}^{t} \mathrm{~d} t^{\prime} \mathcal{K}\left(t, t^{\prime}\right) U_{0 \boldsymbol{k}_{i}}\left(t^{\prime}, t_{0}\right)
\end{aligned}
$$

where

$$
\mathcal{K}\left(t, t^{\prime}\right)=\sum_{i=s, d} \mathcal{D}_{i}\left(t-t^{\prime}\right) V_{\boldsymbol{k}_{i} 0}(t) V_{0 \boldsymbol{k}_{i}}\left(t^{\prime}\right) \exp \left[\mathrm{i} \int_{t^{\prime}}^{t} \mathrm{~d} t^{\prime \prime} \varepsilon_{0}\left(t^{\prime \prime}\right)\right],
$$

and $\mathcal{D}_{i}(t)$ is the Fourier transform of the $i$-th energy band density of states, $\boldsymbol{k}_{i}=$ $k_{s}, \boldsymbol{k}_{d}$ and we assumed that the atom energy level $\varepsilon_{0}$ can be changed along the moving atom trajectory. Note that the information about the $s$ - and d-electron energy bands enters to all functions $U_{00}\left(t, t_{0}\right), U_{0 \boldsymbol{k}_{s}}\left(t, t_{0}\right)$, and $U_{0 \boldsymbol{k}_{d}}\left(t, t_{0}\right)$ through the integral kernel $\mathcal{K}\left(t, t^{\prime}\right)$.

\section{Numerical results and discussion}

In the first step we discuss the problem of the charge transfer between moving atom and substrate surface in the presence of the adsorbed atom. In the second step, at the end of this section, we consider the scattering of atoms on model transition metal surfaces. Equations (21), (25), and (26) have been solved for various 
choices of the surface density of states, $\varepsilon_{0}, \varepsilon_{\mathrm{A}}, \varepsilon_{\mathrm{F}}$ and atom velocities. The required functions $U_{00}\left(t, t_{0}\right), U_{0 a}\left(t, t_{0}\right)$, and $U_{0 k}\left(t, t_{0}\right)$ (see Eq. (8)) have been obtained according to the formulae (19), (23), and (24), respectively, and $n_{0}(t)$ was calculated according to Eq. (8). As mentioned in Sec. 2 we assume the $z$-dependence of the hybridization matrix element $V_{\mathrm{A} 0}(z)$ in the Gaussian form with $\gamma=0.05$ a.u. ${ }^{-2}$ (cf. $[9,11,12,16])$. Our calculations are performed beyond the wide band limit for exactly defined surface density of states. We use the broad and narrow rectangular density of states $\mathcal{D}(E)$ with the bandwidth $D=10 \mathrm{eV}$ and $D=3 \mathrm{eV}$, respectively. In addition, in order to investigate the influence of the shape of $\mathcal{D}(E)$ on the charge transfer we have performed the calculations of $n_{0}$ for the density of states being the superposition of two rectangular parts - the broad part $(10 \mathrm{eV})$ and the narrow part $(1 \mathrm{eV})$ placed at the centre of the broad energy band.

For a better insight into the problem we have also performed calculations for clean surfaces (without adsorbed atoms) and made the comparison with the results obtained for scattering of atoms on atom adsorbed on the surface. We assume that the initial atomic occupancy $n_{0}(0)$ corresponds to negatively ionized atoms and investigate the time evolution of the probability of the negative ionization $n_{0}(t)$ during the scattering on the atom adsorbed on the surface. In other words, we calculate the probability that the negative ionization (the charge state of the moving atom) is not changed in comparison with its initial state.

The final charge state of the scattered atom depends also on the position of the Fermi energy in the band, on the adsorbed atom energy level $\varepsilon_{\mathrm{A}}$, and the moving atom level $\varepsilon_{0}$, and on the atom velocity. In our calculations we place the Fermi energy in different parts of the energy band - in the lower part $\varepsilon_{\mathrm{F}}=-D / 4$, in the middle one of the band $\varepsilon_{\mathrm{F}}=0$, and in the higher part of the band $\varepsilon_{\mathrm{F}}=D / 4$ ( $D$ being the bandwidth and the centre of the band serves as the energy reference point). As for the $\varepsilon_{\mathrm{A}}$ we take it equal to the Fermi energy. In the case of the half-filled energy band $\left(\varepsilon_{\mathrm{F}}=0\right.$ ) this situation corresponds to the neutral state of the chemisorbed atom and for $\varepsilon_{\mathrm{F}}=\mp D / 4$ the adatom is only slightly charged. So, our choice of $\varepsilon_{\mathrm{A}}$ corresponds to neutral or nearly neutral adatom case and, we hope, may be justified for our model calculations. As for $\varepsilon_{0}$, we take it as a constant and calculate the charge state of the moving atom for different values of $\varepsilon_{0}$ extending from bottom to top of the energy band. Finally, we have performed the calculations for two atom velocities, $v=0.02$ a.u. and $v=0.05$ a.u., respectively.

Let us consider the charge transfer dynamics between the moving atom and the surface with adsorbed adatom for the simple case of the empty narrow ( $D=1 \mathrm{eV}$ ) surface energy band and the sudden switching on of the interaction (Fig. 1, thin line). For comparison, we give also $n_{0}(t)$ for an atom scattered on the clean surface (Fig. 1, thick line). In both cases the overall behaviour is similar, we observe the periodic time oscillations, but for scattering on the surface with an adsorbed atom the amplitude of these oscillations is smaller and the charge localized on the moving atom never falls down to zero values. Similar oscillating behaviour 


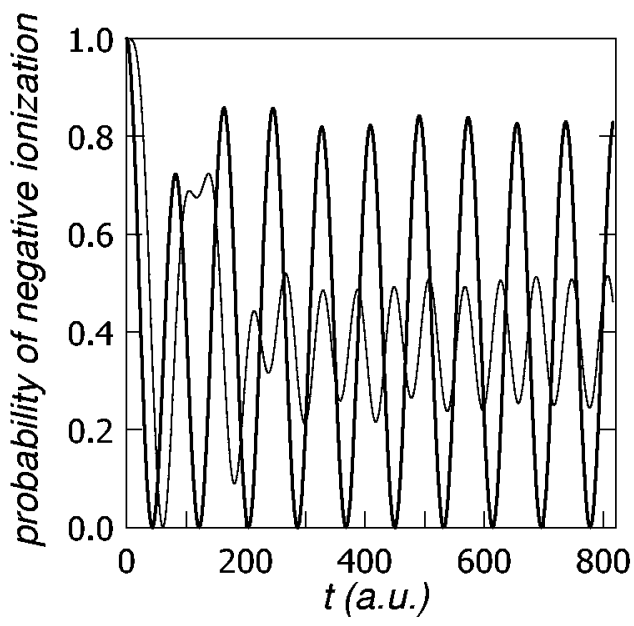

Fig. 1. Time dependence of probability of negative ionization of the atom scattered on a clean surface (thick line) and on a surface with adsorbed atom (thin line). The surface is described by an empty narrow, $D=1 \mathrm{eV}$, rectangular density of states, the energy level of the moving atom $\varepsilon_{0}=0$ and that of the adatom $\varepsilon_{\mathrm{A}}=0$ and the sudden switching on (at time $t=0$ ) of interactions is assumed.

of $n_{0}(t)$ for the case of scattering on clean surfaces was considered and explained by Tsuneyuki et al. in Ref. [1]. The reduced amplitude of the charge oscillations is observed in scattering on the adatom as in this case the electron can be transferred from the atom to the surface (and vice versa) only through the adatom level. Note, however, that the averaged (in time) values of $n_{0}(t)$ are equal in both cases. More complicated dynamics of the charge transfer is obtained from the calculations with realistic $z$-dependencies of the matrix elements $V_{\mathrm{A} 0}$. In Fig. 2 we present $n_{0}(z)$ for the case of scattering on clean (panel $a$ ) and adsorbed (panel $b$ ) surfaces. Here the hybridization matrix elements are taken in the form $V_{\mathrm{A} 0}(z)=V_{\mathrm{A} 0} \exp \left(-\gamma z^{2} / 2\right)$ with $V_{0}=1 \mathrm{eV}$ and $\gamma=0.05 \mathrm{a.u}^{-2}$. The surface is characterised, as in Fig. 1, by the empty narrow $\left(D=1 \mathrm{eV}\right.$ ) electron energy band and $\varepsilon_{0}=\varepsilon_{\mathrm{A}}=0$, and the atom velocity $v$ equals 0.05 a.u. As the atom moves towards the surface, the interaction between it and surface increases and the charge oscillations also increase. The maximal amplitudes of these oscillations are observed near the surface. After collision, on the way outward the surface because of the decreasing $V_{\mathrm{A} 0}(z)$ the charge exchange is smaller and $n_{0}(z)$ is stabilised around the value achieved at some distances from the surface.

Let us note that the minimal values of $n_{0}(z)$ on the way toward and outward the clean surface are equal to zero as in the constant hybridization matrix elements case. A very different picture of the charge transfer dynamics is present for the case of atom scattered on the adatom - Fig. 2b. Similarly to Fig. $1 \mathrm{~b}, n_{0}(z)$ does not attain zero values although oscillates with maximal am- 


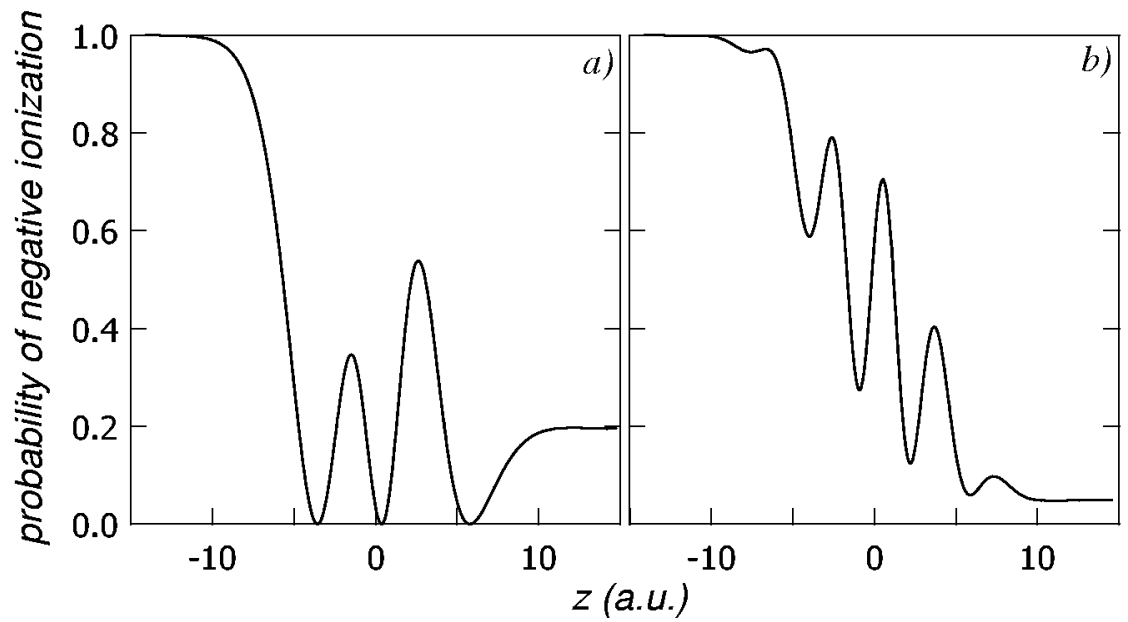

Fig. 2. The same as in Fig. 1 but for $z$-dependent atom-adatom interactions for atom velocity $v=0.05$ a.u. The panel $a(b)$ corresponds to scattering on a clean (with adsorbed atom) surface. The negative $z$ values correspond to the atom moving towards the surface.

plitude at the surface. Note that unexpectedly at the first sight, the asymptotic value $n_{0}(\infty)$ is much smaller than this one for the clean surface case. Because of the impeded transfer of electrons between the moving atom and the surface, one should expect smaller values of $n_{0}(\infty)$ in the clean surface case. However, this is not the case as the asymptotic value $n_{0}(\infty)$ depends on the atom velocity and most of the charge transfer occurs in a relatively small range of distances from the surface. The results shown in Fig. 3 confirm this conclusion. In this

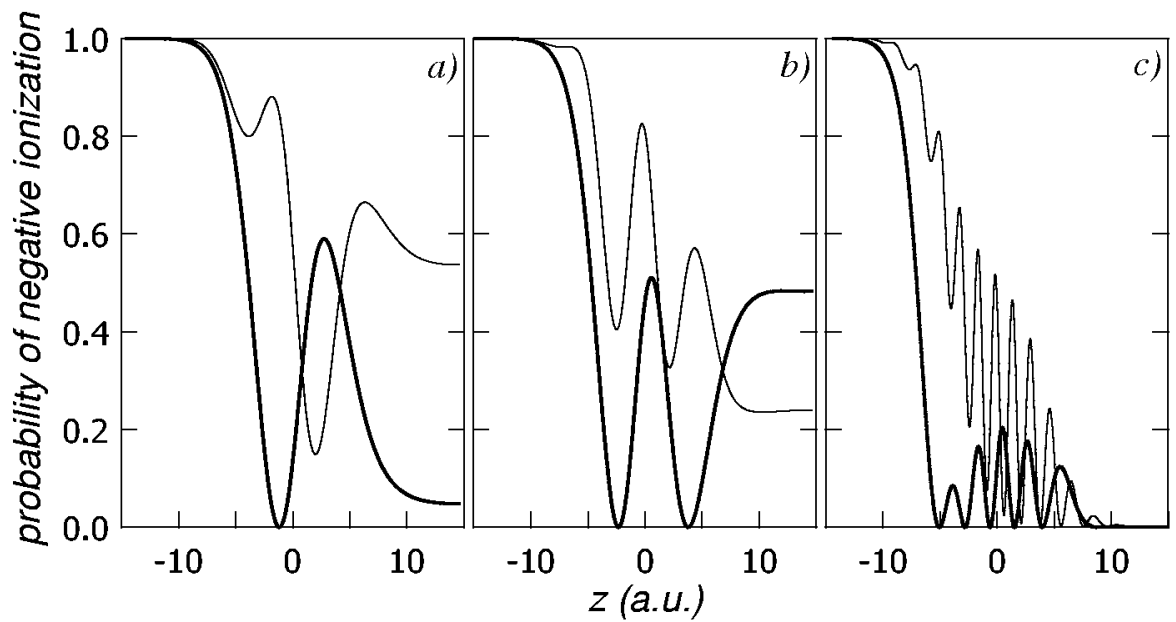

Fig. 3. The same as in Fig. 2 for $v=0.1,0.075$, and 0.025 a.u., panels $a$, $b$, and $c$, respectively. The thick (thin) lines correspond to scattering on a clean (with adsorbed atom) surface. 
figure we present $n_{0}(z)$ for different velocities for scattering on the clean surfaces (thick lines) and on the adatom (thin lines).

In Figs. 4 and 5 we present the charge transfer dynamics for the sudden switching on of the constant $V_{\mathrm{A} 0}$ and for $z$-dependent $V_{\mathrm{A} 0}(z)$, respectively, for clean surfaces (surfaces with adsorbed atom) - panels $a$ (panels $b$ ). The results are obtained for empty surface energy bands and for three types (mentioned earlier) of the density of states. The simplest case for discussion is the one in Fig. 4a. For a broad energy band the electron charge is relatively fast transferred to the surface and $n_{0}(\infty)$ is equal to zero (curve $B$ ). At a smaller width of the energy band $n_{0}(t)$ still relatively fast is falling down at the beginning but oscillates with the constant nonzero amplitude for $t \rightarrow \infty$ (curve $A$ ). Such behaviour was obtained in Ref. [1]. It becomes evident that for the surface characterised by the density of states with some resonance-like structure $n_{0}(t)$ exhibits some features belonging to broad, structureless, as well as, to narrow, resonance-like density of states (curve $C$ ). In this case the ionization probability $n_{0}(t)$ oscillates as for narrow density of states (compare Fig. 1, thick line) and simultaneously decreases as for broad density of states. The case of the charge transfer through the adatom adsorbed on the surface described by different density of states is shown in Fig. 4b. This picture now is more complicated but still it is possible to discuss it in term of the charge transfer dynamics for broad and narrow energy bands.

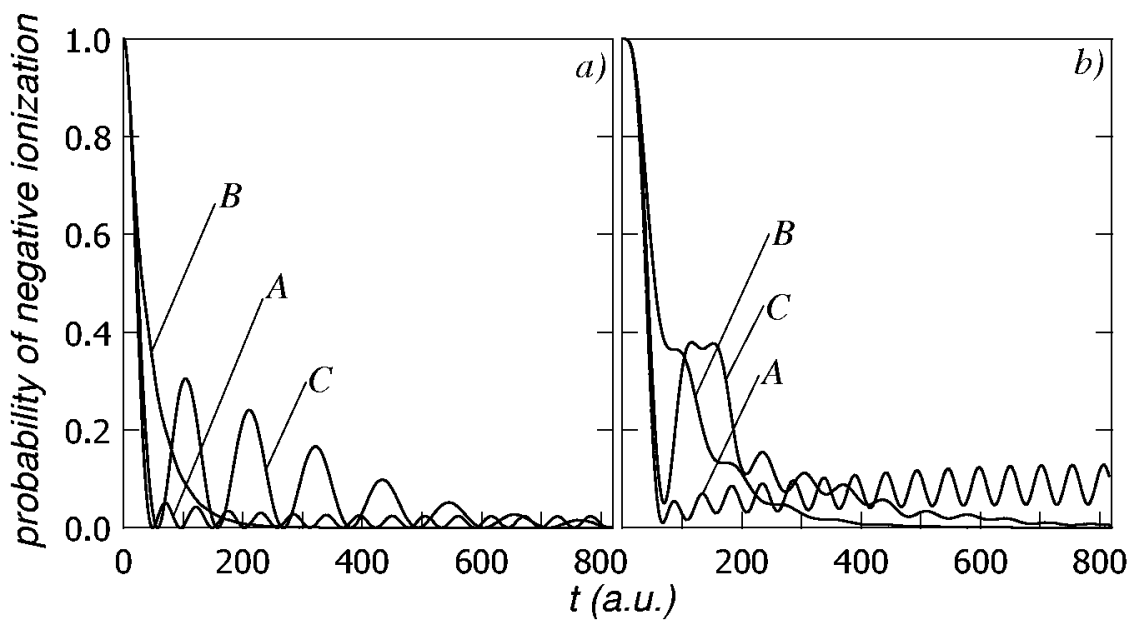

Fig. 4. Time dependence of probability of negative ionization of the atom scattered on a clean surface (panel $a$ ) and on a surface with adsorbed atom (panel $b$ ) for a sudden switching on of the interactions. The curves $A(B)$ correspond to the empty narrow, $D=3 \mathrm{eV}$ (broad, $D=10 \mathrm{eV}$ ) rectangular density of states and the curves $C$ correspond to the broad, $D=10 \mathrm{eV}$, rectangular density of states with an additional, narrow structure in the centre of the band. 
In Figs. 5, 6 we present the results for $n_{0}(\infty)$ as a function of the position of $\varepsilon_{0}$ relative to the band centre. Figure 5 shows the results for clean surface and Fig. 6 for the surface with adsorbed atoms. Curves $A(B)$ correspond to the Fermi energy localised below (above) the band centre, $\varepsilon_{\mathrm{F}}=-D / 4\left(\varepsilon_{\mathrm{F}}=D / 4\right)$. The thick (thin) lines denote the results obtained for the atom velocity $v=0.02(v=0.05)$ a.u. The panels $a, b$, and $c$ correspond to different (mentioned earlier) densities of states.

Let us discuss firstly the case of the clean surface - Fig. 5. For low filling of the surface band $\left(\varepsilon_{\mathrm{F}}=-D / 4\right.$, curves $A$ ) we observe a significant decrease of the negative ionization probability, close to zero values at lower velocities, if the level $\varepsilon_{0}$ moves up from $\varepsilon_{\mathrm{F}}$ towards the band centre. As $\varepsilon_{0}$ moves farther to the upper band limit, $n_{0}(\infty)$ increases and the scattered atom becomes more and more negatively ionized. This picture is valid also for greater bandfillings, $\varepsilon_{\mathrm{F}}=D / 4$, curves $B$. The explanation of such behaviour of $n_{0}(\infty)$ is rather simple. Probability of the negative ionization decreases (the charge is transferred from the moving atom to the surface) if the energy level $\varepsilon_{0}$ is in resonance with empty states of the surface energy band. For $\varepsilon_{0}$ lying close to the upper band limit the probability of the negative ionization grows as the number of empty surface states becomes smaller.

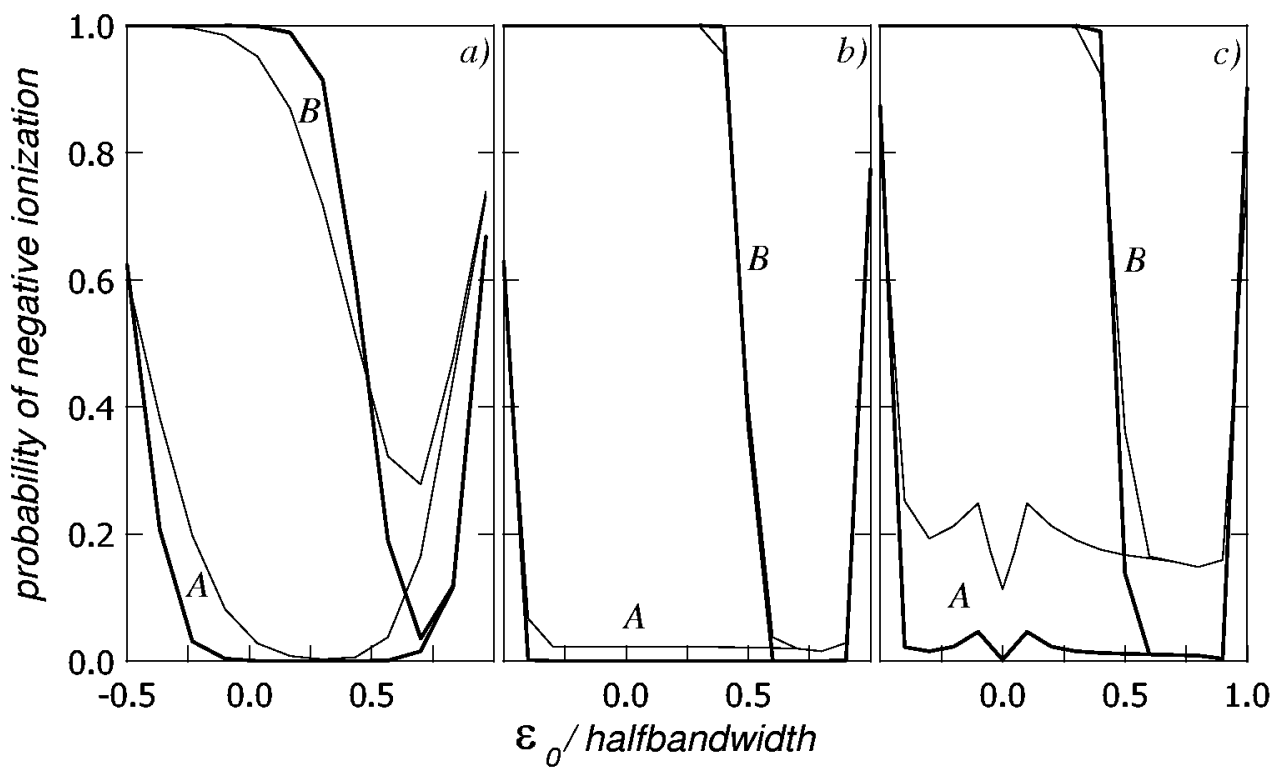

Fig. 5. Probability of the negative ionization vs. atom energy level $\varepsilon_{0}$ for the atoms scattered on the clean surface. The curves $A(B)$ correspond to the Fermi energy equal to $-D / 4(D / 4)-D$ being the bandwidth and zero energy is put at the band centre. The panel $a(b)$ corresponds to the rectangular density of states and $D=3(10) \mathrm{eV}$ and panel $c$ to the rectangular density of states with the peak at the band centre. The thick (thin) lines correspond to atom velocity $v=0.02(0.05)$ a.u. 
More rapidly decreasing values of $n_{0}(\infty)$ for the case of a broad surface energy band (compare curves $A$ in panels $a$ and $b$ in Fig. 5) than for a narrow band can be explained as follows. The electron transferred from the moving atom to a point on the surface escapes from this point within a finite lifetime inversely proportional to the bandwidth. Then, for a wide band probability that the electron comes back to the moving atom is much smaller than for a narrow band (cf. [1]). This picture is valid for small, as well as, for greater atom velocities. In the case of density of states with some resonance-like additional structure at the band centre we observe a clear response of the charge transfer mechanism to such electronic characteristics of the surface. This response is consistent with our earlier considerations - probability of the negative ionization decreases (the charge is transferred from the negative ion to the substrate) for $\varepsilon_{0}$ being in resonance with the enhanced part of the density of accessible empty electron states of the substrate. Note also that the atom velocity is much more important in this case than for structureless density of states.

Let us compare these results with those obtained for moving atoms scattered on the surface with adsorbed atoms $\left(\varepsilon_{\mathrm{A}}=\varepsilon_{\mathrm{F}}\right.$ ) (Fig. 6). The panels $a, b, c$ in Fig. 6 correspond to the same conditions as in Fig. 5 with one exception - now the additional atom is adsorbed on the surface and the moving atom is scattered back from this atom. It is interesting that there are no qualitative differences between the results obtained for the narrow energy band - compare Fig. 5a and Fig. 6a. The essential differences are visible for broad density of states and $\varepsilon_{\mathrm{F}}$ localized in the lower part of the band - compare curves $A$ in Fig. $5 \mathrm{~b}$ and Fig. $6 \mathrm{~b}$. Now

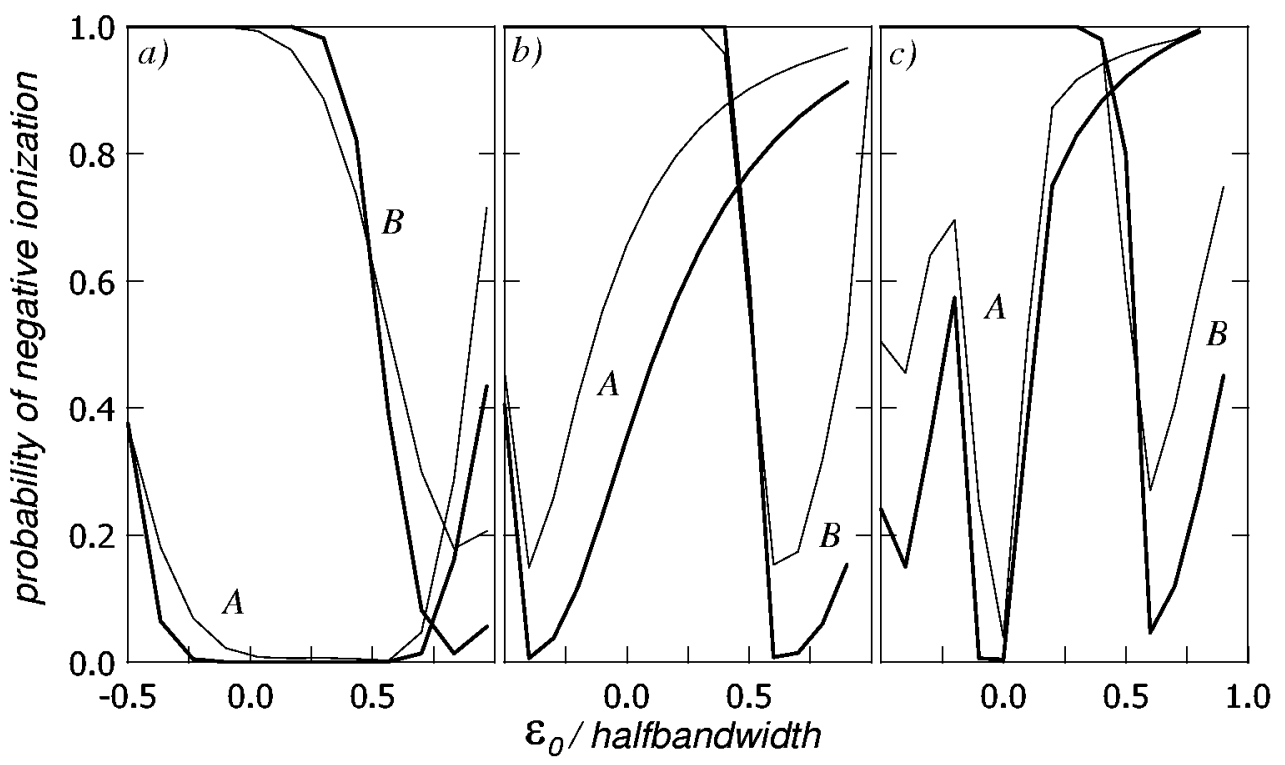

Fig. 6. The same as in Fig. 5 but for moving atom scattered on the surface with adsorbed atom for $\varepsilon_{\mathrm{A}}$ being equal to the Fermi energy. 
the probability of the negative ionization is almost the same as for the clean surfaces case only for $\varepsilon_{0}$ to be near $\varepsilon_{\mathrm{A}}$ and $\varepsilon_{\mathbf{F}}$. For $\varepsilon_{0}$ moving away from $\varepsilon_{\mathbf{A}}$ (and from $\varepsilon_{\mathrm{F}}$ ), the charge transfer between the moving atom and the surface is more and more impeded (Fig. 6b, curves $A$ ). Such behaviour can be easily understood, as the charge transfer can be realized only through the adsorbed atom level and the probability of such process is strongly damped with the increasing difference between $\varepsilon_{0}$ and $\varepsilon_{\mathrm{A}}$. For that reason, there are no essential qualitative differences between the curves $B$ in Fig. $5 \mathrm{~b}$ and $6 \mathrm{~b}$ obtained for $\varepsilon_{\mathrm{F}}$ lying in the upper part of the band $\left(\varepsilon_{\mathrm{F}}=D / 4\right)$. Similarly, for non-constant density of states (Fig. $6 \mathrm{c}$ ) but for $\varepsilon_{\mathrm{F}}$ localized in the upper part of the band (curves $B$ ), the behaviour of probability of negative ionization of the moving atom vs. $\varepsilon_{0}$ does not differ qualitatively from the results for constant surface density of states (compare curves $B$ in Figs. 6b and $6 \mathrm{c}$ ). The only difference are greater minimal values of $n_{0}(\infty)$ in Fig. $6 \mathrm{c}$, which is a consequence of a smaller (in this energy region) density of a vailable electron states for tunneling electrons. The essential differences between results showed in Figs. $6 \mathrm{~b}$ and $6 \mathrm{c}$ are visible for $\varepsilon_{\mathrm{F}}$ (and $\varepsilon_{\mathrm{A}}$ ) lying in the lower part of the density of states below its enhanced part. For $\varepsilon_{0}$ moving from the lower parts of $\mathcal{D}(E)$ up to the enhanced part of $\mathcal{D}(E)$ the behaviour of $n_{0}(\infty)$ is essentially different from this one for constant $\mathcal{D}(E)$. Now for $\varepsilon_{0}$ localized in the energy region of the enhanced part of the surface density of states there is a very large probability of electron tunneling from the moving atom to the surface energy band (a deep minimum on curves $A$, Fig. $6 \mathrm{c}$ ). For greater $\varepsilon_{0}$ localized far away from $\varepsilon_{\mathrm{F}}=\varepsilon_{\mathrm{A}}$ the probability of the negative ionization increases rapidly as for constant $\mathcal{D}(E)$ (compare curves $A$ in Figs. $6 \mathrm{~b}$ and $6 \mathrm{c}$ at higher values of $\varepsilon_{0}$ ).

Now we are going to discuss the charge transfer between the scattered atom and the model transition metals surfaces. In Figs. 7, 8 we present results of the negative ionization probability of atoms scattered on a metal surface described by two overlapped energy bands of different widths. We consider rectangular, broad $(20 \mathrm{eV})$ s-electron-like and narrow $(5 \mathrm{eV}) \mathrm{d}$-electron-like energy bands. In such a manner we simulate the realistic situations of scattering on transition metal surfaces. In these metals there are a broad energy band due to the delocalized $s$-electrons and a narrow energy band corresponding to the localized $d$-electrons. Depending on the kind of metal the narrow $d$-band has a different position relative to the Fermi energy. We have considered two different practical situations. Firstly, we assumed that the narrow band is localized below $\varepsilon_{\mathrm{F}}$ at different distances from it (and is filled). Secondly, the $\varepsilon_{\mathrm{F}}$ crosses the narrow energy band and in this case it is only partially filled. We have calculated the $z$-dependence of the negative ionization probability of atoms for two atom velocities $(v=0.1$ a.u. and $v=0.03$ a.u. $)$ and for low $(\phi=1.5 \mathrm{eV})$ and high $(\phi=3.5 \mathrm{eV})$ work function. In order to come closer to realistic situations we take the $z$-dependence of the atom affinity level in the form $\varepsilon_{0}(z)=-A-1 /\left(z+z_{0}\right)$, where $A$ is the free atom affinity level and $z_{0}$ is a parameter which reduces the image interaction at small distances. 
For $A$ we take $0.7 \mathrm{eV}$ which corresponds to scattering of the negative hydrogen ions. We approximate the $z$-dependence of $V_{0 k_{i}}(z)$ by $V_{0 \boldsymbol{k}_{i}} \exp (-z / \lambda)$, where $\lambda$ is the interaction range (fitted fairly well by $\lambda=2.5$ a.u.) and $V_{0 k_{i}}=2 \mathrm{eV}$.

In Fig. 7 we show $n_{0}(z)$ for the case of filled $d$-bands lying below $\varepsilon_{\mathrm{F}}$. We performed the calculations for different positions of the $d$-band relative to the Fermi energy. We begin from the case, when the upper limit of the band equals to $\varepsilon_{\mathrm{F}}$ and we move down the band up to $2 \mathrm{eV}$ below $\varepsilon_{\mathrm{F}}$. For comparison, the case of the surface characterized by a single, broad energy band is also shown (for a higher velocity, $v=0.1$ a.u.). For a high work function, panel $b$, the picture is rather simple. As the atom affinity level lies above $\varepsilon_{\mathrm{F}}$ on in- and outgoing trajectory, then we observe a relatively large charge transfer from the negative ion to the surface and this transfer hardly depends on the position of the filled $d$-band which entirely is placed below the Fermi energy. Note that the charge transfer to the single $s$-band only is very similar to results obtained for overlapped bands. For a lower work function, panel $a$, the dependence of the charge transfer on the $d$-band position is more clear but the physics of this transfer is similar. Now, for $d$-band states lying closer to the Fermi energy the rate of the neutralization of the negative ions is much smaller in comparison with the case of the band moved down from $\varepsilon_{\mathrm{F}}$.

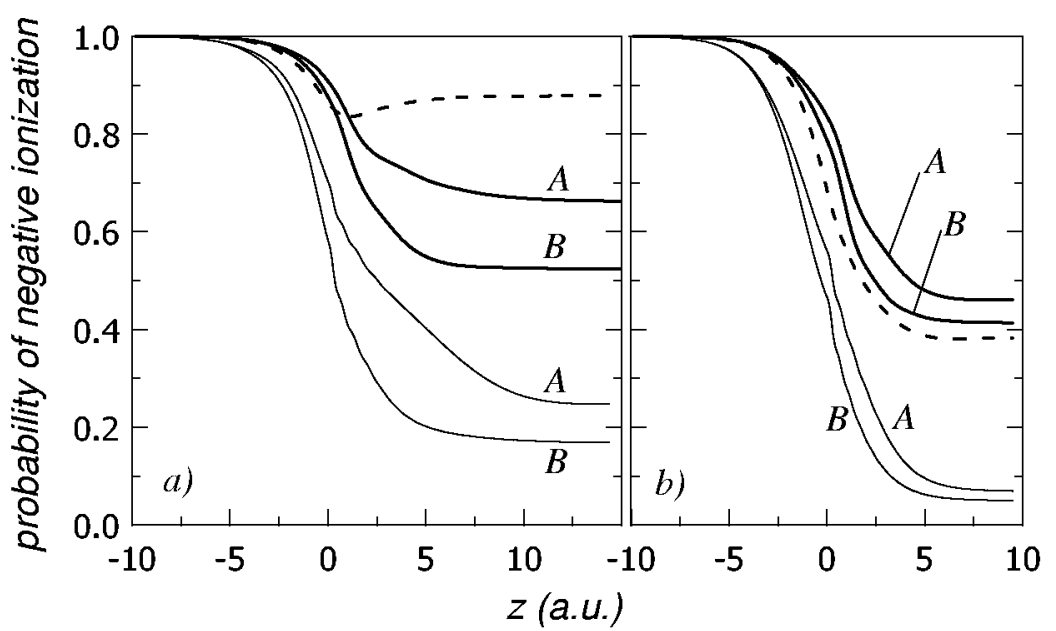

Fig. 7. The $z$-dependence of the probability of negative ionization of the atom scattered on the clean substrate characterised by two overlapped energy bands of width $20 \mathrm{eV}$ and $5 \mathrm{eV}$. The panel $a(b)$ corresponds to the work function $1.5 \mathrm{eV}(3.5 \mathrm{eV})$ and the thick (thin) curves correspond to the atom velocity $v=0.1$ a.u. $\left(0.03\right.$ a.u.). $V_{0 \boldsymbol{k}_{i}}=2 \mathrm{eV}$, $\lambda=2.5$ a.u., $z_{0}=3$ a.u., and $A=0.7 \mathrm{eV}$. The upper limit of the $d$-electron energy band is equal to $\varepsilon_{\mathrm{F}}$ (curves $A$ ) and is placed $2 \mathrm{eV}$ below $\varepsilon_{\mathrm{F}}$ (curves $B$ ). For comparison, broken curves correspond to the substrate described by $s$-band only $(v=0.1$ a.u.). 


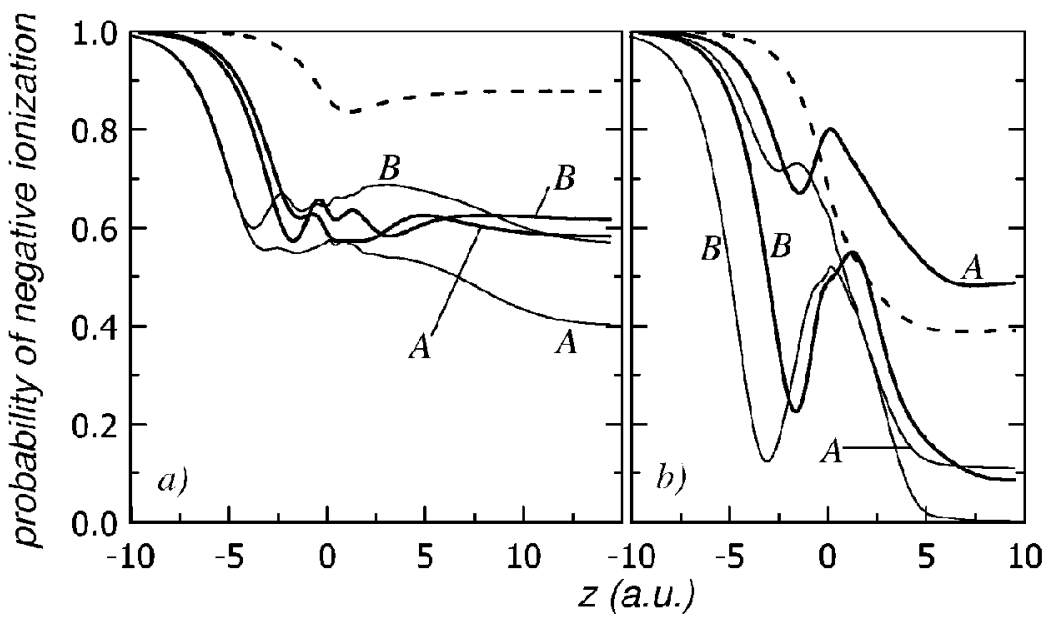

Fig. 8. The same as in Fig. 7 but for partially filled $d$-electron energy band. The lower limit of this band is localised $3.5 \mathrm{eV}(1.5 \mathrm{eV})$ below $\varepsilon_{\mathrm{F}}-$ curves $A(B)$.

Let us consider now the case of partially filled $d$-bands, Fig. 8 . For the higher work function, panel $b$, the charge transfer depends on the $d$-band filling - for nearly filled $d$-bands $n_{0}(\infty)$ is much greater than for smaller fillings. In the second case the electrons can be transferred between ion and empty $s$ - and $d$-bands (we remember that $\varepsilon_{0}(z)>\varepsilon_{\mathrm{F}}$ for all atom trajectory), whereas and in the first case the empty $d$-band electron states are available for ion electron only at the smallest distance from the surface. On the other hand, for the lower work function (panel $a$ ), especially at a higher atom velocity, these differences are small. Now for small, as well as, for large band fillings $n_{0}(z)$ are comparable.

In conclusion, the effect of the metal band characteristics on the resonant charge transfer in ion - adsorbed atom collision was theoretically studied based on the Anderson-Newns Hamiltonian. We used the narrow and broad rectangular density of states and also the density of states with a sharp structure inside the band. The case of negative ions was considered and probability of the negative ionization was calculated for various combinations of the Fermi energy and positions of the moving and adsorbed atom energy levels and for different ion velocities. The main conclusions can be summarized as follows:

- Depending on the ion velocity, the charge transfer from the moving ion to the substrate through the adsorbed atom level can be enhanced, as well as damped in comparison with scattering on clean surfaces (Fig. 3).

- The charge transfer between the moving negative ion and the surface is similar for clean and adsorbate-covered surfaces for narrow energy bands (Figs. 5a and 6a) (irrespective of the Fermi energy and localization of $\varepsilon_{0}$ $\left.\left(\varepsilon_{\mathrm{F}}=\varepsilon_{\mathrm{A}}\right)\right)$. 
- For broad substrate energy bands probability of the negative ionization of the moving atom strongly depends on the relative position of $\varepsilon_{0}$ and $\varepsilon_{\mathrm{A}}$. For $\varepsilon_{\mathrm{F}}$ lying in the lower part of the band (curve $A$, Fig. $6 \mathrm{~b}$ ) and for $\varepsilon_{0}$ moving from the bottom of the band approximately up to $\varepsilon_{\mathrm{A}}\left(\varepsilon_{\mathrm{A}}=\varepsilon_{\mathrm{F}}\right)$ probability of the negative ionization decreases rapidly and then increases as $\varepsilon_{0}$ moves up to the upper band limit. In this point there are significant differences in comparison with the clean surface case for which probability of the negative ionization for $\varepsilon_{0}>\varepsilon_{\mathrm{F}}$ is still small until $\varepsilon_{0}$ comes nearly to the upper band limit. The above conclusions are valid for both small and higher ion velocities. For $\varepsilon_{\mathrm{F}}$ lying in the upper part of the band (compare curves $B$ in Figs. 5b and $6 \mathrm{~b}$ ) there are no qualitative differences between scattering on clean surfaces or on the atom adsorbed on the surface.

- In the case of scattering of negative ions on the clean metal surfaces characterised by overlapping broad and narrow energy bands the charge transfer hardly depends on the position of the filled narrow band (relative to the Fermi energy) for the higher work function (Fig. 7b). For the low work function this dependence is more visible (Fig. 7a). In the case of partially filled narrow band the dependence of the charge transfer on the band position relative to $\varepsilon_{\mathrm{F}}$ is sharply outlined for higher work functions (Fig. 8b), whereas for the low work function this dependence is less visible (especially for higher atom velocities).

\section{References}

[1] S. Tsuneyuki, N. Shima, M. Tsukada, Surf. Sci. 186, 26 (1987).

[2] M. Tsukada, Progr. Theor. Physics Suppl. 106, 257 (1991).

[3] M. Tsukada, N. Shima, in: Dynamical Process and Ordering on Solid Surfaces, Eds. A. Yoshimori, M. Tsukada, Springer Verlag, Berlin 1995, p. 34.

[4] K.L. Sebastian, V.C.J. Bhasu, T.B. Grimley, Surf. Sci. 110, L571 (1981).

[5] T.B. Grimley, V.C.J. Bhasu, K.L. Sebastian, Surf. Sci. 121, 305 (1983).

[6] M. Kato, D.J. O'Connor, K. Yamamoto, R. Souda, Surf. Sci. 363, 150 (1996).

[7] A. Yoshimori, K. Makoshi, Progr. Surf. Sci. 21, 25 (1986).

[8] R. Brako, D.M. Newns, Surf. Sci. 108, 253 (1981).

[9] H. Kasai, A. Okiji, Surf. Sci. 183, 147 (1987).

[10] R. Taranko, Phys. Status Solidi B 199, 3939 (1997).

[11] R. Taranko, E. Taranko, Vacuum 54, 161 (1999).

[12] R. Taranko, E. Taranko, Surf. Sci. 441, 167 (1999).

[13] M. Wiertel, R. Taranko, E. Taranko, Acta Phys. Pol. A 96, 769 (1999).

[14] Y. Muda, D.M. Newns, Phys. Rev. B 37, 7048 (1988).

[15] H.K. McDowell, J. Chem. Phys. 77, 3263 (1982). 
[16] M.C.G. Passeggi, E.C. Goldbeerg, J. Ferron, Phys. Rev. B 35, 8330 (1987).

[17] J. Morino, N. Lorente, F. Flores, M.Yu. Gusev, Nucl. Instrum. Methods, Phys. Res. B 125, 289 (1997).

[18] M.Yu. Gusev, D.V. Klushin, I.F. Urazgildin, S.V. Sharov, J. Exp. Theor. Phys. 103, 2102 (1993) (in Russian).

[19] W. Bloss, D. Hone, Surf. Sci. 72, 277 (1978).

[20] J.J.C. Geerlings, R. Rodink, J. Los, J.P. Gauyacq, Surf. Sci. 186, 15 (1987).

[21] H. Nakanishi, H. Kasai, A. Okiji, Surf. Sci. 197, 515 (1988).

[22] E.G. Overbsch, J. Los, Surf. Sci. 108, 117 (1989).

[23] J.P. Gauyacq, A.G. Borisov, J. Phys., Condens. Matter 10, 6585 (1988).

[24] G.A. Kimmel, D.M. Goodstein, B.H. Cooper, J. Vac. Sci. Technol. A 7, 2186 (1989).

[25] C.B. Weare, J.A. Yarmoff, Surf. Sci. 348, 359 (1996).

[26] C.B. Weare, J.A. Yarmoff, J. Vac. Sci. Technol. A 13, 1421 (1995).

[27] J.A. Yarmoff, C.B. Weare, Nucl. Instrum. Methods Phys. Res. B 125, 262 (1997). 